\section{Neu auf dem Markt}

Das Unternehmen neuraxpharm hat seine ZNS-Palette erweitert. Ab sofort sind Naratriptan-neuraxpharm ${ }^{\circledast}$ und Zolmitriptanneuraxpharm ${ }^{\circledast}$ zur akuten Behandlung der Migräne mit oder ohne Aura verfügbar. Zolmitriptan-neuraxpharm ${ }^{\circledast}$ gibt es in den Wirkstärken $2,5 \mathrm{mg}$ und $5 \mathrm{mg}$ und Naratriptan-neuraxpharm ${ }^{\circledast}$ in der Wirkstärke 2,5 mg. Außerdem ist ab sofort Ropinirolneuraxpharm ${ }^{\circledast}$ in einer Retard-Darreichung verfügbar. Neben den schnell freisetzenden Filmtabletten gibt es Ropinirolneuraxpharm ${ }^{\circledast}$ nun in den Retard-Wirkstärken $2 \mathrm{mg}, 3 \mathrm{mg}, 4 \mathrm{mg}$ und $8 \mathrm{mg}$. red

Nach Informationen von neuraxpharm

\section{Multiple Sklerose}

Die dreimal wöchentliche subkutane Gabe von Glatirameracetat $40 \mathrm{mg} / 1 \mathrm{ml}$ senkt bei Patienten mit schubförmig remittierender Multipler Sklerose die jährliche Schubrate im Vergleich zu Placebo signifikant um 34,4\%, so das Ergebnis der Phase-III-Studie GALA (Glatirameracetat LowFrequency Administration). Damit wurde der primäre Endpunkt der Studie erreicht. An der einjährigen randomisierten, doppelblinden, placebokontrollierten Studie nahmen mehr als 1.400 Patienten an 155 Standorten in verschiedenen Ländern teil. Eine erste Auswertung der Daten weist darauf hin, dass auch die sekundären klinischen Endpunkte erreicht wurden - mit Ausnahme der Verringerung der Hirnatrophie. Die in GALA verabreichte Dosis von $40 \mathrm{mg} / 1 \mathrm{ml}$ ist höher als die derzeit vermarktete tägliche Dosis von $20 \mathrm{mg} / 1 \mathrm{ml}$ Glatirameracetat (Copaxone ${ }^{\circledast}$ ).

Nach Informationen von Teva

\section{Interaktive Online-Fortbildung}

Begeben Sie sich in einer interaktiven Online-Fortbildung zusammen mit dem Medizindetektiv Dr. Wolgemuth auf Spurensuche im Fall einer Depression mit körperlichen Symptomen. Der Fall: Julia Müller war lebenslustig und erfolgreich, bis sie durch eine Depression antriebslos wurde und Rückenschmerzen und Beklemmungen in der Brust bekam. Auch die Behandlung mit einem Trizyklikum ändert daran wenig und führt dazu, dass sie zunimmt und ständig müde ist. Lösen Sie den Fall unter www.lillyfortbildung.de. red

Nach Informationen von Lilly

\section{MS-induzierte Spastik: Mehr als ein Drittel erhält keine antispastische Medikation}

Nach den Ergebnissen der deutschen Querschnittsstudie MOVE 1 leiden mehr als zwei Drittel der Patienten mit Multipler Sklerose (MS) dauerhaft unter Spastizität. Bei drei Vierteln war die Symptomatik laut Arzturteil mittelschwer oder schwer ausgeprägt, am häufigsten waren die Beine betroffen. Zwar wurde die Mehrzahl (60,9\%) der Patienten mit antispastischen Medikamenten behandelt, Patienten und Ärzte waren mit der Wirksamkeit aber oftmals nicht zufrieden.

Die wichtigsten Ergebnisse aus MOVE 1 stellte Professor Peter Flachenecker, Chefarzt des Neurologischen Rehabilitationszentrums Quellenhof in Bad Wildbad, vor. Er betonte, dass MS-induzierte Spastiken die Aktivitäten des täglichen Lebens erheblich beeinträchtigen.

Grundlage der Studie waren retrospektiv erhobene Daten aus den vergangenen zwölf Monaten sowie das Arzturteil und Patientenangaben bei Visiten zu verschiedenen Zeitpunkten. An der Studie nahmen 414 Patienten an 42 Zentren teil. Die Patienten waren im Mittel 48,6 $( \pm 9,6)$ Jahre alt und im Schnitt $13,9( \pm 8,6)$ Jahre an MS erkrankt. Die Patienten litten im Mittel seit $8,2( \pm 5,9)$ Jahren an Spastiken. 68,6\% der Patienten hatten dauerhaft spastische Symptome, bei $28,7 \%$ waren diese schwer, bei 44,0\% mittelschwer und bei 27,3\% leicht ausgeprägt. Am häufigsten waren die Beine betroffen (56,3\% der Patienten mit Paraspastik).
Als am stärksten beeinträchtigende Symptome der Spastik nannten die Patienten Muskelsteifigkeit (74,4\%), eingeschränkte Mobilität $(66,2 \%)$, Erschöpfung/Fatigue $(48,1 \%)$, Blasenstörungen $(45,2 \%)$ und Schmerzen (44,4\%). Diese Einschätzung deckte sich in hohem Maße mit dem Arzturteil.

Zunehmende Spastizität war mit einem höheren Behinderungsgrad und geringerer Lebensqualität korreliert. Insgesamt wurden $60,9 \%$ der Patienten medikamentös antispastisch behandelt, 37,2\% nicht. Sogar Patienten mit schwerer Spastik erhielten zu $16,9 \%$ keine antispastische Medikation. Patienten und Ärzte waren mit der Wirksamkeit der antispastischen medikamentösen Therapie mehrheitlich nicht oder nur teilweise zufrieden (Abbildung 1).

Die MOVE 1-Studie spiegelt Art, Merkmale und Belastung durch die Spastizität bei MSPatienten vor der Einführung des Endocannabinoidsystem-Modulators Sativex ${ }^{\oplus}$ (Wirkstoffe: Delta-9-Tetrahydrocannabinol, THC, und Cannabidiol, CBD) wider. Die derzeit laufende Studie MOVE 2 soll die Situation nach der Einführung ermitteln.

Dr. Gunter Freese

Fachpresse-Roundtable „MS-induzierte Spastik: der Patient im Fokus", Hamburg, 31.5.2012; Veranstalter: Almirall

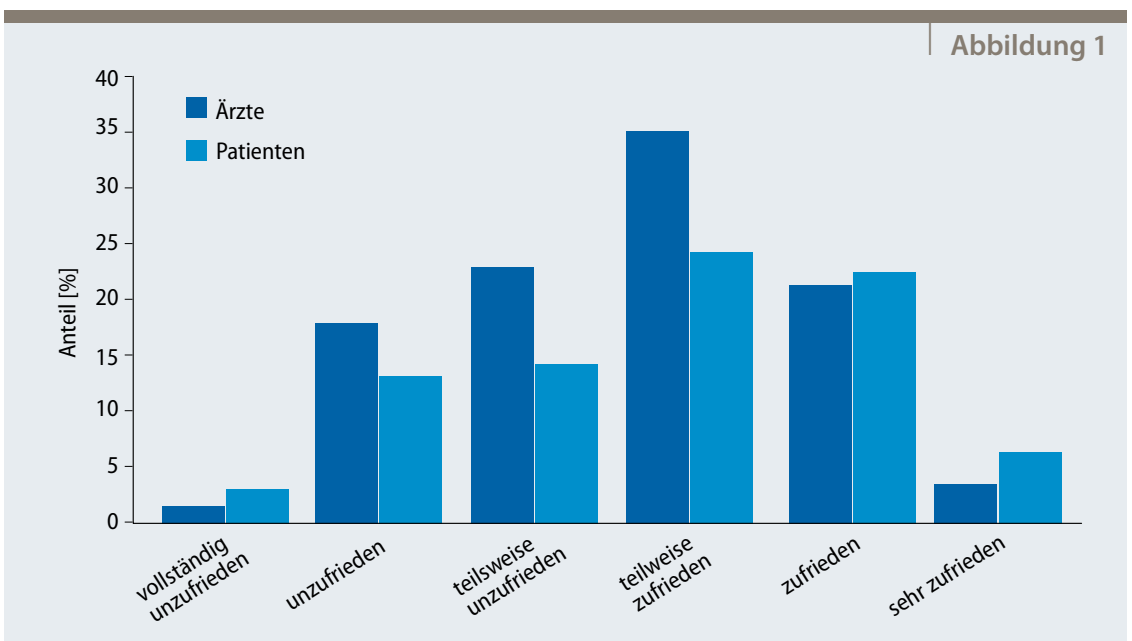

Zufriedenheit mit der derzeitigen antispastischen Medikation im Arzt- und Patientenurteil (MOVE 1-Studienergebnisse; Vortrag Professor Flachenecker). 